\title{
Performance Function for Time-Jittered Equispaced Sampling Wattmeters
}

\author{
Domenico Mirri, Gaetano Iuculano, Gaetano Pasini, and Fabio Filicori
}

\begin{abstract}
This paper evaluates the effect of time-jitter in the equally spaced sampling wattmeters on the hypothesis of equal effects in the two channels and a jitter uncorrelated with the input signals. It is shown that time-jitter, which is a random fluctuation with respect to the nominal sampling time, introduces a frequency limitation which is evaluated together with that due to the sampling strategy and filtering algorithm. The theoretical results are compared with the simulated ones.
\end{abstract}

\section{INTRODUCTION}

$\mathbf{R}$ ANDOM fluctuation with respect to the nominal sampling instant is commonly called timing-jitter. Its effect on the amplitude and phase estimates of sampled signals [1], [2] and on the recovery of a signal from noise by averaging [3] has been studied by different authors. This paper investigates the effect of time-jitter in digital wattmeters based on equally spaced sampling techniques on the hypothesis of equal random fluctuations in the voltage and current channels and a jitter uncorrelated with the input signals. Section II summarizes the properties of the equally spaced synchronous, quasisynchronous and asynchronous sampling strategies in terms of accuracy and bandwidth [4], [5]. Section III quantifies the effect of time-jitter and expresses the estimate of the output as a function of the sampling instants. Section IV gives the expressions of the asymptotic parameters which characterize the estimate of the output of the instrument [6], [7] and compares the theoretical values with the experimental results.

\section{Equally SPACEd SAMPling StRategies}

Let us assume that the instantaneous power $p(t)$ is periodic with period $T_{1}$; therefore it can be represented by the Fourier series:

$$
p(t)=\sum_{q=-\infty}^{+\infty} P_{q} e^{j 2 \pi q f_{1} t}=P_{0}+\sum_{\substack{q=-\infty \\ q \neq 0}}^{+\infty} P_{q} e^{j 2 \pi q f_{1} t}
$$

where: $f_{1}=1 / T_{1}, P_{-q}=P_{q}^{*}$, and $P_{0}$ is the mean value of $p(t)$, i.e., the value of the measurand. The distribution of power in the signal $p(t)$ as a function of frequency can be deduced from the power density spectrum $S_{p}(f)$ :

$$
S_{p}(f)=\sum_{q=-\infty}^{+\infty}\left|P_{q}\right|^{2} \delta\left(f-q f_{1}\right)
$$

where $\delta(f)$ is the Dirac function.

Manuscript received May 9, 1994; revised February 8, 1995.

D. Mirri, G. Pasini, and F. Filicori are with Facoltà di Ingegneria, 40136 Bologna, Italy.

G. Iuculano is with Facoltà di Ingegneria, 50139 Firenze, Italy. IEEE Log Number 9411475.
For the digital implementation of a wattmeter, the instantaneous power $p(t)=v(t) i(t)$ is evaluated, at any discrete time instant $t_{i}$, by multiplying the sampled values of the instantaneous voltage and current. On the hypothesis of an adequate resolution, the digital multiplication does not introduce any relevant error, and the sampling procedure can be assumed directly effected on the instantaneous power when the main aim is the theoretical study of the errors due to the sampling strategy and the filtering algorithm.

The sampling techniques here considered are of an equally spaced type, i.e., any sampling instant $t_{i}$ differs from the preceding one by a constant lag $T_{c}$ :

$$
t_{i}=t_{i-1}+T_{c}=\tau_{0}+i T_{c}
$$

where $i$ is an integer, $T_{c}$ is the sampling interval, and $\tau_{0}$ is the shift between the initial sampling instant and the time origin of $P_{0}$. In the synchronous case $\tau_{0}$ is related to the instantaneous power $p(t)$ through the synchronizing circuit, while in the asynchronous one it is independent.

The discrete output of the instrument is an estimate of $P_{0}$ and can be deduced by forming a weighted average of the last $N$ successive values of the instantaneous power (finite impulse response filter, FIR):

$$
\hat{P}_{k}=\sum_{i=0}^{N-1} a_{i} p\left(t_{k-i}\right)
$$

where each output is marked with an integer $k$. The estimate $\hat{P}_{k}$ continuously changes in an asynchronous sampling strategy, while it remains unchanged in the synchronous one $\left(\hat{P}_{k}=\hat{P}\right)$ because the $N$ sampling instants of any successive average are always the same.

By substituting (1) and (3) into (4) we can write:

$$
\begin{aligned}
\hat{P}_{k} & =\sum_{q=-\infty}^{+\infty} P_{q} \sum_{i=0}^{N-1} a_{i} e^{j 2 \pi q f_{1} t_{k-i}} \\
& =P_{0} \sum_{i=0}^{N-1} a_{i}+\sum_{\substack{q=-\infty \\
q \neq 0}}^{+\infty} P_{q} e^{j 2 \pi q f_{1}\left(\tau_{0}+k T_{c}\right)} H\left(q f_{1} T_{c}\right)
\end{aligned}
$$

where $H\left(f T_{c}\right)$ is the frequency response of the finite impulse response (FIR) filter used; this response is periodic of period $f_{c}$. In order to obtain a scale factor equal to one in (5), the sum of the $N$ coefficients $a_{i}$ must be equal to one. Obviously the coefficients $a_{i}$ must be selected to obtain a frequency response which adequately attenuates the contribution to $\hat{P}_{k}$ of each 
spectral component of $p(t)$. By assuming $a_{i}=1 / N$ (rectangular window) we obtain the well-known Dirichlet-Kernel formula:

$$
H\left(f T_{c}\right)=e^{-j \pi f T_{c}(N-1)} \frac{\operatorname{sinc}\left(f N T_{c}\right)}{\operatorname{sinc}\left(f T_{c}\right)}
$$

which is a periodic function assuming a value equal to one for $f T_{c}$ integer and, in the first period, values equal to zero for $f T_{c}=i / N$, with $i=0,1 \cdots N-1$. Therefore, by using a synchronous sampling strategy in which $f_{1} T_{c}$ coincides with one of the zeroes of $H\left(f T_{c}\right)$ :

$$
f_{1}^{\prime} T_{c}=\frac{r}{N} .
$$

Selecting $r$ as a positive integer having no common submultiple with $N$, only the spectral components of $p(t)$ multiple integer of $N$ contribute to the output [4], i.e., when $q=z N$ ( $z$ integer) in (5):

$$
\hat{P}=P_{0}+\sum_{\substack{z=-\infty \\ z \neq 0}}^{+\infty} P_{z N} e^{j 2 \pi z r T_{0} f_{c}} .
$$

The output becomes independent from $k$ and the bandwidth, relative to $p(t)$, results therefore in $(N-1) f_{1} . T_{c}$ and $N$ in (7) can be selected independently provided that the signals do not change in the observation (or summation) interval $N T_{c}$, i.e., the interval in which the $N$ samples are taken, the interval which is equal to an integer number of periods $T_{1}\left(N T_{c}=r T_{1}\right)$.

By imposing for every $q \neq z N$ the condition

$$
\left|H\left(q f_{1} T_{c}\right)\right| \leq \frac{1}{N}
$$

the contribution to $\hat{P}_{k}$ of the corresponding spectral components is not null as in the synchronous sampling strategy, but it can be assumed negligible if $N$ is adequately high. Equation (8) still being approximately valid, this asynchronous sampling strategy has been called quasisynchronous. It can be shown that $(9)$ is satisfied when:

$$
\left|q N f_{1} T_{c}-\left\langle q N f_{1} T_{c}\right\rangle\right| \leq\left|q f_{1} T_{c}-\left\langle q f_{1} T_{c}\right\rangle\right|
$$

where the symbol $\langle x\rangle$ means the nearest integer to $x$. On the hypothesis of $f_{1} T_{c}<0.5$, the condition relative to the fundamental component $(q=1)$ becomes [5]:

$$
\left|N T_{c}-r_{1} T_{1}\right| \leq T_{c} \quad \text { for } f_{1} T_{c}<0.5
$$

where $r_{1}$ is the nearest integer to $N T_{c} / T_{1}$. This condition must be compared with that relative to the synchronous sampling strategy (7). In the quasisynchronous case $f_{1}$ and $T_{c}$ are uncorrelated; therefore (7) cannot be satisfied. We can nevertheless conveniently adjust $N$ so that the observation interval $N T_{c}$ becomes as close as possible to an integer number of periods $T_{1}$. Once $N$ is fixed, the bandwidth is evaluated determining the minimum value of $q$ which does not satisfy (10).

In the asynchronous case the bandwidth is instead limited by the periodicity of the function $H\left(f T_{c}\right)$, i.e., $f_{c}$, because the product $f T_{c}$ can assume any value. Therefore the FIR filter must be selected in such a way to minimize $\left|H\left(f T_{c}\right)\right|$ within this interval.

\section{THE EFFECT OF TIMING JitTER IN THE EOUALly SPACED SAMPLING STRATEgIES}

Time-jitter introduces random fluctuations with respect to the nominal sampling instants. On the hypothesis of common random fluctuations in the voltage and current channels, the effect of these fluctuations can be analyzed by adding to the second member of (3) the quantity $X_{i} T_{c}$, where $X_{i} T_{c}$ is the $i$ th deviation from the nominal sampling time (the random variables are written in boldface). The output of the instrument can therefore be evaluated by assuming in (4):

$$
\boldsymbol{t}_{k-i}=\boldsymbol{\tau}_{0}+\left(k-i+\boldsymbol{X}_{k-i}\right) T_{c} .
$$

The nuisance or incidental parameter $\boldsymbol{X}_{k-i}$ is the $i$ th of a set of independent random variables having a continuous distribution with characteristic function $\Phi\left(f T_{c}\right)$, null mean value and variance $\sigma^{2}$.

A generic discrete output of the instrument is identified by the labeling mark $k$, an integer which can be considered randomly picked up from a sequence of $2 h+1$ possible successive values $(-h \leq k \leq+h)$, each one of the outputs having an equal chance of being selected [6], [7]. In the synchronous case the shift $\tau_{0}$ depends on the synchronizing circuit; in the asynchronous one, instead, it is strictly related to the turn-on instant of the instrument. In both cases $\tau_{0}$ is a priori unknown, and its actual value can be considered a realization of a continuous set of values that are equally likely to fall anywhere within some generic time interval $(-T / 2,+T / 2, T$ being unknown). Therefore we introduce $\tau_{0}$ as a continuous random variable uniformly distributed in a time interval $T$ and $\boldsymbol{k}$ as a discrete random variable uniformly distributed in the interval $2 h+1$. Obviously, the variability of the output of the instrument when the sampling strategy is synchronous without time-jitter is due solely to the hypothesized variability of $\tau_{0}$ [7]

When each random variable $\boldsymbol{X}_{\boldsymbol{i}}$ is expressly generated by the instrument with a continuous uniform distribution in an interval $\pm T_{c} / 2$, (12) describes a random sampling strategy whose properties were previously studied by the authors [8].

\section{Performance ANALysis}

The output quantity $\hat{P}_{k}=f\left(\tau_{0}, \underline{X}_{k}\right)$ is a function of the random variables $\boldsymbol{k}, \boldsymbol{\tau}_{0}$ and the vector $\underline{X}_{k}$ of the $N$ random variables $\boldsymbol{X}_{\boldsymbol{k}_{-i}}$ used to obtain the output labeled with $k$. An appropriate characterization of the output uncertainty can be obtained by evaluating the statistical parameters of $\hat{P}_{\boldsymbol{k}}$, i.e., the mean value $M\left\{\hat{P}_{\boldsymbol{k}}\right\}$ and the mean square error $M\left\{\left(\hat{P}_{k}-P_{0}\right)^{2}\right\}$. In order to incorporate all the a priori chances into the instrument's performance, in the asynchronous case the number of the output states, i.e., $2 h+1$ must be sufficiently large and theoretically tend to infinity; in the synchronous one it is the excursion of the initial shift $\tau_{0}$, i.e., $T$, which must instead tend to infinity [7]. Therefore, in both cases, we consider the asymptotic statistical parameter, i.e., the asymptotic mean $\bar{P}=\underline{M}\left\{\hat{P}_{k}\right\}$ and the asymptotic mean square error:

$$
E^{2}=\underset{\rightarrow}{M}\left\{\left(\hat{P}_{\boldsymbol{k}}-P_{0}\right)^{2}\right\}=\underset{\rightarrow}{M}\left\{\hat{P}_{\boldsymbol{k}}^{2}\right\}-2 P_{0} \underset{\rightarrow}{M}\left\{\hat{P}_{\boldsymbol{k}}\right\}+P_{0}^{2}
$$


On the hypothesis of time-jitter uncorrelated with the input signals, it can be shown [7], [8] that $\bar{P}=P_{0} \sum_{i=0}^{N-1} a_{i}$ and:

$$
E^{2}=2 P_{0}^{2}\left[1-\sum_{i=0}^{N-1} a_{i}\right]+2 \sum_{q=1}^{+\infty}\left|P_{q}\right|^{2} W^{2}\left(q f_{1} T_{c}\right) \text {. }
$$

The first term of the second member represents the asymptotic bias, and the second one the asymptotic variance. On the hypothesis that $\sum_{i=0}^{N-1} a_{i}=1$, the output of the instrument is asymptotically unbiased, and the asymptotic mean square error coincides with the asymptotic variance. In this hypothesis, by referring to (2), (14) can be rewritten as follows:

$$
E^{2}=\int_{-\infty}^{+\infty} S_{p}(f) W^{2}\left(f T_{c}\right) d f-P_{0}^{2}
$$

with $W^{2}(0)$ being equal to 1 . This equation shows that the asymptotic mean square error can be obtained by multiplying the power density spectrum of $p(t)$, excluding the dc component, with the function $W^{2}\left(f T_{c}\right)$ which weights the contribution of each spectral component of the power density spectrum; for this reason we call $W^{2}\left(f T_{c}\right)$ the weighting function. Therefore the behavior of this last as a function of frequency completely describes the performance of the instrument in terms of accuracy and bandwidth.

By assuming the FIR filter is fitted with a rectangular window with $a_{i}=1 / N(6)$, the weighting function can be written as follows [7], [8]:

$$
\begin{aligned}
W^{2}\left(f T_{c}\right)= & \frac{1}{N}\left(1-\left|\Phi\left(f T_{c}\right)\right|^{2}\right) \\
& +\left|\Phi\left(f T_{c}\right)\right|^{2} \frac{\operatorname{sinc}^{2}\left(N f T_{c}\right)}{\operatorname{sinc}^{2}\left(f T_{c}\right)} .
\end{aligned}
$$

For time-jitter-free sampling, $|\Phi|$ is equal to one and consequently, $W^{2}=\operatorname{sinc}^{2}\left(N f T_{c}\right) / \operatorname{sinc}^{2}\left(f T_{c}\right)$. With timejitter present, $|\Phi|$ becomes less than one and a function of frequency; therefore, the first term of the sum in (16) results not null and variable with frequency while, in the second term, the effect of the digital filter is attenuated. When jitter is uniformly distributed in the range $\pm b T_{c}$, we have $\Phi\left(f T_{c}\right)=\operatorname{sinc}\left(2 b f T_{c}\right)$. Fig. 1 shows the weighting functions of the equally spaced sampling strategies without jitter (dashed line) and with a jitter uniformly distributed with $\pm b= \pm 0.1$ (continuous line); the value $N=10$ was selected uniquely to make clearer the different shapes of the two functions. This figure shows that time-jitter leads to values of the relative minima increasing with frequency and values of the relative maxima decreasing with frequency.

In an equally spaced synchronous sampling strategy the second term of the sum in (16) is null for $q \neq z N$, while the first one, in the presence of time-jitter, is not null; in this case the contribution of the spectral components for which the condition $q \neq z N$ is verified must also be investigated to limit the asymptotic mean square error. Therefore, by imposing a maximum value of the weighting function, an upper limit of the frequency of the wattmeter can be deduced from (16) or Fig. 1, once the jitter distribution is fixed.

A synchronous wattmeter was simulated by assuming $f_{1}=$ $100 \mathrm{~Hz}$ and $T_{c}=20 \mu \mathrm{s}$. From (7) we deduce $N=500$

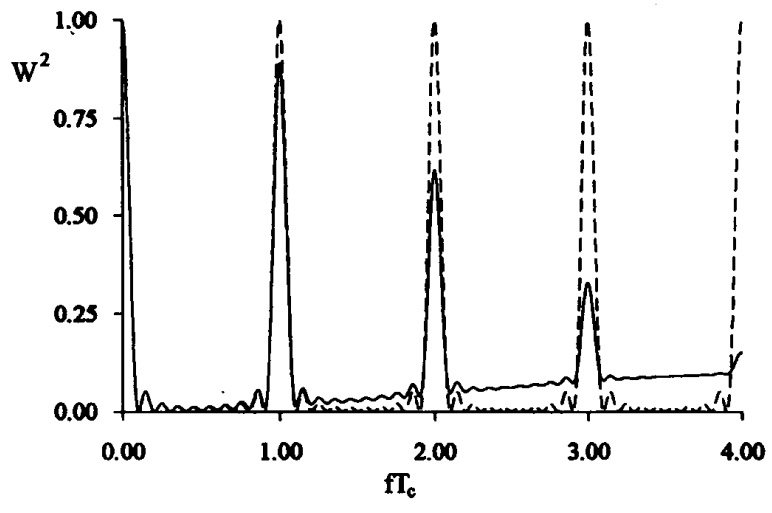

Fig. 1. Weighting function of a synchronous sampling strategy without jitter (dashed line) and jitter uniformly distributed in the interval $\pm b T_{c}= \pm 0.1 T_{c}$ (continuous line) as a function of $f T_{c}(N=10)$.

and $r=1$; therefore only the harmonic components of $p(t)$ at frequencies equal to or multiple integer of $N f_{1}=50$ $\mathrm{kHz}$ contribute to the mean square error. With a time-jitter present, the weighting function must also be investigated at the other harmonic components. On the hypothesis of a uniform distribution with $\pm b= \pm 0.01$, the upper limit of the frequency $\left(f_{\max }\right)$ for two prefixed values of the root of the weighting function $\left(10^{-3}\right.$ and $\left.10^{-4}\right)$ was theoretically deduced from (16). By assuming an instantaneous power with the unique harmonic component at frequency $f_{\max }$ of amplitude equal to the fundamental one, the asymptotic values of $P_{0}$ and $W$ were estimated by considering fifty different outputs and comparing with the theoretical values (Table I).

Due to time-jitter, the sampling instants are randomly generated; therefore the accuracy can be increased with additional measurements. Table I also gives the results when the summation intervals are multiple integers of the previous one. For $N \geq 4 \cdot 500$ and $W=10^{-3}$ the upper limit of frequency is imposed by the second term in (16), instead of the first one as in the previous cases. Obviously, this limit can be overcome by selecting a different value of $T_{c}$ so that $r$ can be an integer greater than one in (7). The experimental results were in very good agreement with the theoretical ones.

\section{CONCLUSIONS}

This paper evaluated the effect of time-jitter in the equally spaced sampling wattmeters on the hypothesis of equal random fluctuations in the voltage and current channels and a jitter uncorrelated with the input signals. After a brief overview of the properties of the synchronous, quasisynchronous and asynchronous sampling strategies in terms of uncertainty and bandwidth, the effect of time-jitter, which introduces random fluctuations with respect to the nominal sampling instants, was investigated. The asymptotic variance of the output was deduced, and it was shown that the instrument's accuracy can be described by a function which weights each component of the power density spectrum of the instantaneous power. This function, depending on the variance of the random variable associated with the time-jitter and on the number of the sampling instants in the summation interval, allows 
TABLE I

Comparison Between THeoretical Values and THeir ASYMPTOTIC ESTIMATES IN A TIME-JITTERED SYNCHRONOUS SAMPLING WATTMETER $\left(T_{c}=20 \mu \mathrm{s}\right.$ WheN $\left.f_{1}=100 \mathrm{~Hz}\right)$

\begin{tabular}{|c|c|c|c|c|c|}
\hline $\mathrm{N}$ & $\mathrm{W}$ & $\mathrm{f}_{\max }(\mathrm{kHz})$ & $\Delta \mathrm{W}=\mathrm{W}_{\mathrm{fmax}}-\mathrm{W}_{\mathrm{fmax}}$ & $\Delta \mathrm{P}_{0}=\mathrm{P}_{0}-\hat{\mathrm{P}}_{0}$ & $\hat{\mathrm{P}}_{0}$ \\
\hline 500 & $1 \cdot 10^{-3}$ & 30.8 & $-1.8 \cdot 10^{-5}$ & $3 \cdot 10^{-3}$ & 1.00003 \\
& $1 \cdot 10^{-4}$ & 3.0 & $2.5 \cdot 10^{-7}$ & $-4 \cdot 10^{-6}$ & 0.999996 \\
\hline \multirow{2}{*}{$2 \cdot 500$} & $1 \cdot 10^{-3}$ & 43.5 & $-7.0 \cdot 10^{-7}$ & $3 \cdot 10^{-3}$ & 1.00003 \\
& $1 \cdot 10^{-4}$ & 4.3 & $-1.7 \cdot 10^{-6}$ & $9 \cdot 10^{-7}$ & 1.0000009 \\
\hline \multirow{2}{*}{$4 \cdot 500$} & $1 \cdot 10^{-3}$ & 49.9 & $-1.0 \cdot 10^{-5}$ & $2 \cdot 10^{-5}$ & 1.000002 \\
& $1 \cdot 10^{-4}$ & 6.1 & $-4.7 \cdot 10^{-6}$ & $-9 \cdot 10^{-7}$ & 0.9999991 \\
\hline \multirow{2}{*}{$10 \cdot 500$} & $1 \cdot 10^{-3}$ & 49.9 & $3.0 \cdot 10^{-6}$ & $1 \cdot 10^{-7}$ & 1.0000001 \\
& $1 \cdot 10^{-4}$ & 9.7 & $2.0 \cdot 10^{-6}$ & $-2 \cdot 10^{-6}$ & 0.999998 \\
\hline \multirow{2}{*}{$20 \cdot 500$} & $1 \cdot 10^{-3}$ & 49.9 & $-3.6 \cdot 10^{-6}$ & $8 \cdot 10^{-6}$ & 1.000008 \\
& $1 \cdot 10^{-4}$ & 13.7 & $-5.3 \cdot 10^{-6}$ & $-1 \cdot 10^{-6}$ & 0.999999 \\
\hline
\end{tabular}

the bandwidth due to time-jitter to be deduced, both in the synchronous and quasisynchronous sampling strategies.

The experimental results, obtained with a simulated wattmeter, confirmed the validity of the proposed analysis.

\section{ACKNOWLEDGMENT}

The authors are indebted to Prof. I. Kollár, Technical University of Budapest, who suggested extending our studies on the random sampling techniques to the time-jitter effect, and to Prof. U. Tiberio, of the University of Florence, for the algebraic suggestions.

\section{REFERENCES}

[1] T. M. Sunders, D. R. Flach, C. Hagwood, and G. L. Yang, "The effects of timing jitter in sampling systems," IEEE Trans. Instrum. Meas., vol. 39 , no. 1 , pp. $80-85$, Feb. 1990.

[2] S. S. Award and M. F. Wagdy, "More on jitter effects on sinewave measurements," IEEE Trans. Instrum. Meas., vol. 40, no. 3, pp. 549-552, June 1991.

[3] M. Sun, D. L. Bonaddio, J. Mi, and R. J. Sclabassi, "An analysis of the effects of jitter in data acquisition on synchronous averaging " IEEE Trans. Syst, Man, Cybern, vol 21, no 2, pp. 456-463, Mar/Apr. 1991.

[4] F. J. J. Clarke and J. R. Stockton, "Principle and theory of wattmeters operating on the basis of regularly spaced sample pairs," J. Phis. E: Sc. Instr., vol. 15, pp. 645-652, 1982.

[5] G. N. Stenbakken, "A wideband sampling wattmeter," IEEE Trans. Power Appar. Syst., vol. PAS-103, no. 10, pp. 2919-2926, Oct. 1984.

[6] F. Filicori, G. Iuculano, A. Menchetti, D. Mirri, and M. Catelani, "New performance function for the comparison of different sampling strategies in nonlinear conversion instruments," in Proc. IEEE Instrum. Meas. Techn. Conf., Washington, Apr. 1989, pp. 307-311.

[7] G. Iuculano, D. Mirri, F. Filicori, A. Menchetti, and M. Catelani, "A criterion for the analysis of asynchronous and synchronous sampling instruments based on non linear processing," IEE Proc. A, vol. 139, no. 4, pp. 141-152, July 1992

[8] F. Filicori, G. Iuculano, A. Menchetti, and D. Mirri, "A random asynchronous sampling strategy for measurement instrument based on nonlinear signal conversion," IEE Proc., vol. 136, pt. A, no. 3, pp. 141-150, May 1989.

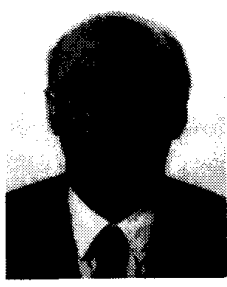

Domenico Mirri was born in Italy in 1936 . He graduated in electronic engineering at the University of Bologna.

$\mathrm{He}$ is Professor of Electrical Measurement. His main areas of interest are now power and biomedical measurements, digital signal processing, and real time analysis.

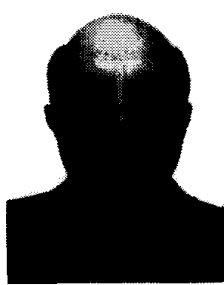

Gaetano Iuculano received the degree in electronic engineering.

At present he is Full Professor of Electrical Measurements and Metrology at the University of Florence. His current research interests are calibration applications, reliability analysis and life testing for electronic devices and systems, statistical analysis, and digital measurement instruments.

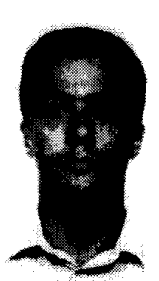

Gaetano Pasini was born in Italy in 1964. He graduated in electronic engineering at the University of Bologna where now is Researcher in Electrical Measurement

His research activity is mainly oriented to digital signal processing and power measurements.

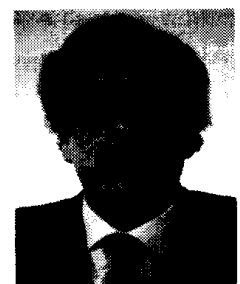

Fabio Filicori was born in Italy in 1949. He graduated in electronic engineering at the University of Bologna in 1974

At present he is Full Professor at the University of Bologna. His current research interest is in the areas of nonlinear circuit analysis and design, electronic devices modeling, digital measurement instruments, and power electronics. 